\title{
Positive feedback between ROS and cis-axis of PIASxa/p38a- SUMOylation/MK2 facilitates gastric cancer metastasis
}

\author{
Qian Wang ${ }^{1,4}, C i X^{1,4}$, Qiang Fan ${ }^{1,4}$, Haihua Yuan ${ }^{1}$, Xin Zhang ${ }^{2}$, Biying Chen ${ }^{1}$, Renjie Cai ${ }^{1}$, Yanjie Zhang $\mathbb{D}^{1,3 凶}$, Moubin Lin $\mathbb{D}^{2 凶}$ and \\ Ming $\mathrm{Xu}$ iD ${ }^{1 凶}$
}

(c) The Author(s) 2021

\begin{abstract}
MAPK/p38 is an important mammalian signaling cascade that responds to a variety of intracellular or extracellular stimuli, such as reactive oxygen species (ROS), and participates in numerous physiological and pathological processes. However, the biological function of p38 in different tumors, and even at different stages of the same tumor, remains elusive. To further understand the regulatory mechanism of p38 and oxidative stress in the occurrence and development of gastric cancer, we report SUMOylation as a novel post-translational modification occurring on lysine 152 of MAPK14/p38a through immunoprecipitation and series of pulldown assays in vitro and in vivo. Importantly, we determine that p38a-SUMOylation functions as an authentic sensor and accelerator of reactive oxygen species generation via interaction with and activation of MK2 in the nucleus, and the ROS accumulation, in turn, promotes the SUMOylation of p38a by stabilizing the PIASxa protein. This precise regulatory mechanism is exploited by gastric cancer cells to create an internal environment for survival and, ultimately, metastasis. This study reveals novel insights into p38a-SUMOylation and its association with the intracellular oxidative stress response, which is closely related to the processes of gastric cancer. Furthermore, the PIASxa/p38a-SUMOylation/MK2 cis-axis may serve as a desirable therapeutic target in gastric cancer as targeting PIASxa, MK2, or a specific peptide region of $\mathrm{p} 38 \mathrm{a}$ may reconcile the aberrant oxidative stress response in gastric cancer cells.
\end{abstract}

Cell Death and Disease (2021)12:986; https://doi.org/10.1038/s41419-021-04302-6

\section{INTRODUCTION}

The cellular response to extracellular stimuli is, in part, dynamically mediated by a number of intracellular kinases and phosphatases [1, 2]. As members of discrete signaling cascades, mitogen-activated protein kinases (MAPKs) transduce signals from the cell membrane to the nucleus in response to diverse extracellular stimuli and regulate fundamental cellular processes [2], as well as pathological courses $[3,4]$. The p38 mitogenactivated protein kinase (MAPK/p38) signaling pathway is involved in a variety of biological phenomena, especially oxidative stress (OS) and DNA damage, which are important causative factors of carcinogenesis [5-9].

Gastric cancer (GC) is the fifth most prevalent malignancy globally and is the third leading cause of cancer-related deaths [10]. H. pylori infection is the most common risk factor for GC, and stepwise progression of this cancer often leads to atrophic gastritis or intestinal metaplasia [10,11]. Although chemotherapy can partially improve the survival and quality of life of patients, especially those with locally unresectable or metastatic GC, the median overall survival is still only around one year. This is because GC is frequently diagnosed at an advanced stage, wherein distant metastases may have occurred [10, 12].
Despite advances in the general understanding of the biology of GC, surgical or endoscopic resection remains the primary treatment strategy for GC [11].

The progression of GC is a complex process with multiple steps and stages, all of which involve DNA damage, excessive proliferation, apoptotic damage, and gene instability [10, 13]. Cellular signaling pathways altered in GC cells include the NF-KB, ERK/MAPK, ROS/ASK1/JNK, and MAPK/p38 pathways [14, 15]. Recent studies have shown that OS in cells is closely related to the occurrence and development of cancers [16]. Low concentrations of ROS in the tumor microenvironment can promote tumor glucose metabolism by inducing mitochondrial autophagy and activating signaling pathways to maintain the demand for high consumption of energy in tumors [16]. Persistently high ROS levels can cause oncogene activation, gene mutations, or chromosomal aberrations $[17,18]$. Contrastingly, high ROS concentrations can also inhibit the growth and development of GC cells by inducing tumor cell apoptosis [19-21]. However, in-depth understanding of the effect of oxidative stress on the occurrence and development of gastric cancer is still in puzzle.

ROS are a class of oxidizing agents (including $\mathrm{O}_{2}{ }^{-}, \mathrm{OH}, \mathrm{H}_{2} \mathrm{O}_{2}$, $\mathrm{NO}$, and $\mathrm{ONOO}^{-}$) that exert diverse effects on cellular components

\footnotetext{
'Department of Oncology, Shanghai Ninth People's Hospital, Shanghai Jiao Tong University School of Medicine, 280 Mohe Road, Shanghai 201999, China. ${ }^{2}$ Center for Clinical Research and Translational Medicine, Yangpu Hospital, Tongji University School of Medicine, 16 Boyang Road, Shanghai 200090, China. ${ }^{3}$ Shanghai Institute of Precision Medicine, Ninth People's Hospital, Shanghai Jiao Tong University School of Medicine, 115 Jinzun Road, Shanghai 200125, China. ${ }^{4}$ These authors contributed equally: Qian Wang, Ci Xu, Qiang Fan. ${ }^{凶}$ email: zhangyanjie@shsmu.edu.cn; 1500142@tongji.edu.cn; mingxu.msu@gmail.com Edited by Professor Nickolai Barlev
}

Received: 15 April 2021 Revised: 5 October 2021 Accepted: 11 October 2021 Published online: 22 October 2021 
and play critical roles in maintaining tissue homeostasis [22-24]. Notably, high ROS levels can be reversed by corresponding cellular antioxidants (SOD, CAT, GSH-Px, SRX, TXN, vitamin C/E, and $\mathrm{Zn}^{2+}$ ) to prevent adverse consequences and to ensure an intracellular balance, which has a great impact on determining malignant progression of cancers [24-26]. In cancer cells, p38a is a key OS sensor in oncogenic transformation, and its activation can significantly inhibit HRAS $^{\mathrm{V} 12}$-induced ROS accumulation by triggering apoptosis. Thus, p38a could function as a negative modulator of tumor initiation or malignant transformation under OS [9]. Interestingly, ROS was found to promote the proliferation of pancreatic ductal adenocarcinoma cells by inducing monocyteto-myofibroblast trans-differentiation through the MAPK/ p38 signaling pathway [27]. The activation of MAPK/p38 limits the lifespan of hematopoietic stem cells by increasing ROS levels [6]; however, this increase induced protective autophagy through regulation of autophagy-related genes in HeLa cells [28]. Therefore, the role of activation or inhibition of MAPK/p38 in promoting human cancer progression in the presence of OS largely depends on the tumor type or stage. However, there are still undiscovered regulatory mechanisms between OS and p38 that contribute to the occurrence and development of GC.

SUMOylation, one of the most common type of reversible posttranslational modifications (PTMs), is essential for the maintenance of genomic integrity, gene regulation, and intracellular signaling through dynamic regulation of protein structure, interaction, and spatial localization via attachment of small ubiquitin-like modifier (SUMO) to the lysine $\varepsilon$ amino group or the SUMO interaction motif (SIM) $[29,30]$. Additionally, SUMOylation levels on target proteins can be influenced by diverse intracellular or extracellular stimuli, such as growth factors [31, 32], inflammatory cytokines [33], kinase activities [34], hypoxia contexts [35], and ROS levels [34, 36, 37]. Thus, imbalance of the intracellular SUMO system or SUMOylation levels of functional substrates is closely related to disease development, including tumor initiation and cancer metastasis [30].

In this study, we reveal that p38a can be covalently SUMOylated which is associated with cellular OS. Therefore, understanding how OS responding and ROS production are precisely regulated during the development of GC and how p38a-SUMOylation is utilized to maintain the survival and metastasis of tumor cells under the ROS circumstance is of great clinical significance for the development of new strategies for the prevention and treatment of this cancer disease.

\section{MATERIALS AND METHODS \\ SUMOylation assays}

SUMOylation assay was performed according to the three different methods as described in previous reports [38-40]. Detailed procedures are available from supplemental materials.

\section{ROS detection}

Intracellular ROS levels were measured with fluorescent probes $2^{\prime}, 7^{\prime}$ dichlorofluorescein diacetate (DCFH-DA) and CellROX Orange Reagent as per the manufacturer's instructions. Detailed procedures are available from supplemental materials.

\footnotetext{
Animal xenograft assay

After pre-selection (weight, size, or healthy state), four-week-old female nude mice $(B A L B / C)$ were divided into four groups randomly. The mice were bred in a specific pathogen-free condition in which the temperature was maintained at $22-25^{\circ} \mathrm{C}$ and the humidity was set at $40 \%$. Mice were injected with $1 \times 10^{6}$ cells in $100 \mu \mathrm{L}$ PBS via tail vein. A total of 5 mice in each group were monitored every three days and sacrificed at 45 days post injection. Lungs and livers of mice were collected and fixed with $4 \%$ paraformaldehyde for blind hematoxylin and eosin ( $H$ \& E) and immunohistochemistry $(\mathrm{IHC})$ staining analysis. The strong vimentin staining in IHC was considered the positive tumor loci. The experiment
}

was approved by the Animal Ethics Committee of Shanghai Jiao Tong University School of Medicine.

\section{Statistical analysis}

Each experiment was performed at least three times. The significance of the difference and variation between any two non-paired samples was calculated and tested with a $t$-test by using GraphPad Prism software (GraphPad Software, San Diego, CA, USA), in which $p<0.05\left(^{*}\right)$ or $<0.01(* *)$ was considered statistically significant.

\section{RESULTS}

\section{SUMOylation occurs on p38a in the nucleus}

To determine whether p38a was SUMOylated, the $\mathrm{Ni}^{2+}-\mathrm{NTA}$ pull-down assay was conducted. The results showed that HisSUMO1 conjugated with HA-p38a (Fig. 1a, left panel), or FlagSUMO1 conjugated with His-p38 (Fig. 1a, right panel), and the reaction could be enhanced by SUMOylation E2 enzyme Ubc9 or attenuated by de-SUMOylation enzyme SENP1 in HEK293T cells, respectively (Fig. 1a and Fig. S1a). A similar result was observed by an immunoprecipitation assay performed according to a previous report [40] (Fig. 1b and Fig. S1b). Furthermore, a GST pulldown assay using an $E$. coli prokaryotic expression system confirmed that GST-p38a was strongly SUMOylated upon cotransformation with pE1/E2/SUMO1 (Fig. 1c and Fig. S1c). To identify endogenous SUMOylation on p38a, lysates of MGC803 and HGC27 cell lines were analyzed by an immunoprecipitation assay. Endogenous SUMO1modified p38a was only successfully detected by $\mathrm{p} 38 \mathrm{a}$ antibody in the complexes immunoprecipitated with the SUMO1 antibody but not in those immunoprecipitated with the normal lgG (Fig. 1d). A similar phenomenon was also observed by analyzing the lysates of tumor and adjacent surgical tissues from GC patients (Fig. 1e). Moreover, the SUMOylation on p38a occurred only in the nucleus according to $\mathrm{Ni}^{2+}-\mathrm{NTA}$ pull-down assay by using the extracted lysates of nuclear/cytosol fractionation (Fig. 1f). Collectively, these results demonstrated that p38a can be SUMOylated in vitro and in vivo, and this modification takes place only in the nucleus.

\section{SUMO1 is mainly attached to K152 in p38a}

According to the prediction by JASSA (http://www.jassa.fr) and GPS-SUMO 2.0 Online Service (http://sumosp.biocuckoo.org/) (Fig. $1 \mathrm{~g}$ and Fig. S1d, e), a total of six potential SUMOylation sites were selected within $\mathrm{p} 38 \mathrm{a}$ and mutated by replacing $\mathrm{R}$ (arginine) with $\mathrm{K}$ (lysine). Wild-type (WT) or mutant HA-p38a (K53R, K54R, K79R, K165R, K152R, K267R) constructs were cotransfected with His-SUMO1 into HEK293T cells for the $\mathrm{Ni}^{2+}$ NTA pull-down assay. We found that the K152R mutation reduced the SUMOylation level of p38a, but the other mutants exhibited no or weak changes compared with those of $\mathrm{p} 38 \mathrm{a}^{\mathrm{WT}}$ (Fig. 1h). Immunoprecipitation assay also confirmed that K152 was the major SUMO1 modification site in p38a (Fig. 1i). However, K152 has already been reported as the acetylation site in p38a [41]. To exclude the impact of acetylation on p38a in follow-up experiments, we tried to disrupt the canonical consensus motif of $\psi$-K-x-D/E (directionless; $\psi$, a hydrophobic amino acid; $x$, any amino acid residue; $D_{150} L_{152} P$ in $p 38 a$ ) with D150A or D150S for exclusively affecting SUMOylation, without potentially impairing acetylation of p38a. $\mathrm{Ni}^{2+}-\mathrm{NTA}$ pull-down and immunoprecipitation assays showed that D150A or D150S, both similar to K152R, could reduce p38a-SUMOylation level (Fig. 2a, b), but barely affected its acetylation level (Fig. 2c, d). Together, these results suggested that K152 is a major SUMO1 modification site in $\mathrm{p} 38 \mathrm{a}$, and $\mathrm{p} 38 \mathrm{a}$ variants harboring a mutation of D150A or D150S can be further used for analyzing the exact function of SUMOylation in p38a, without interference or 'noise' derived from acetylation. 


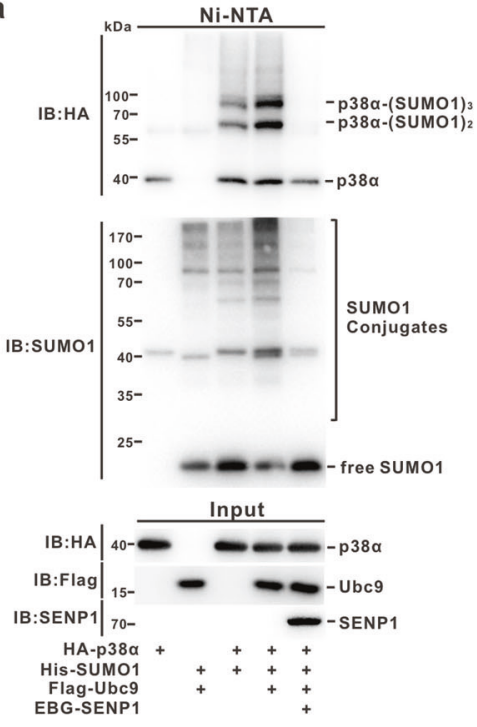

c

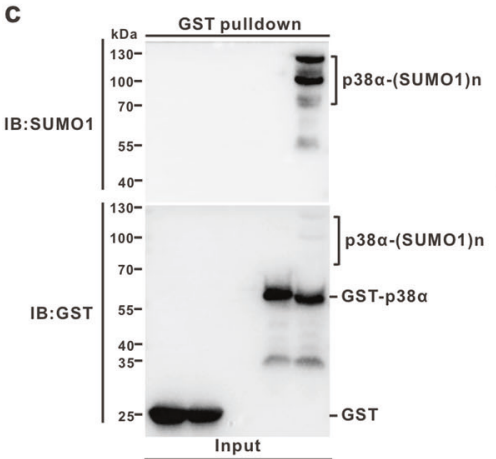

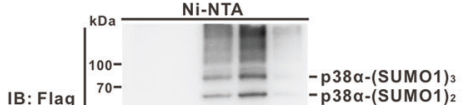

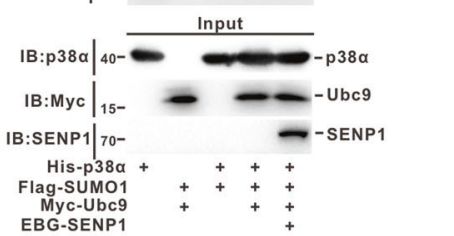

d

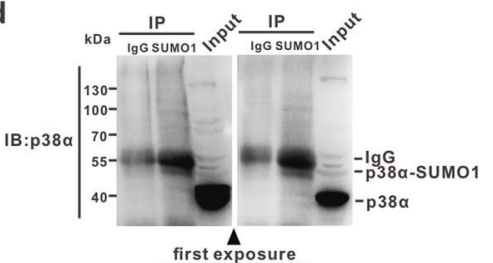

b
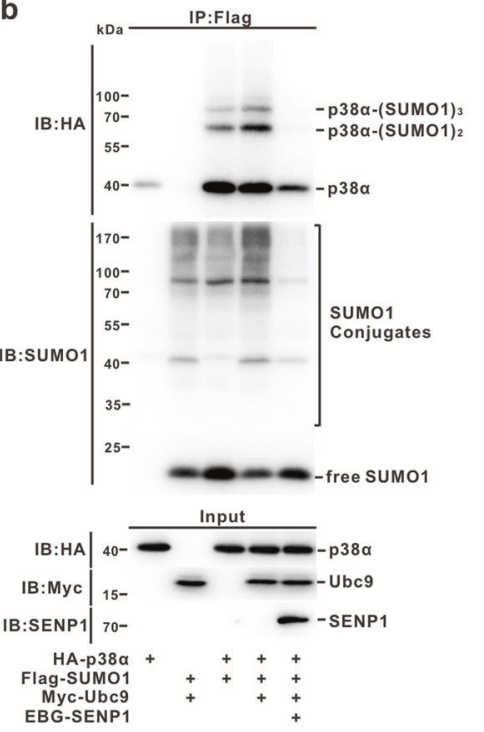

e

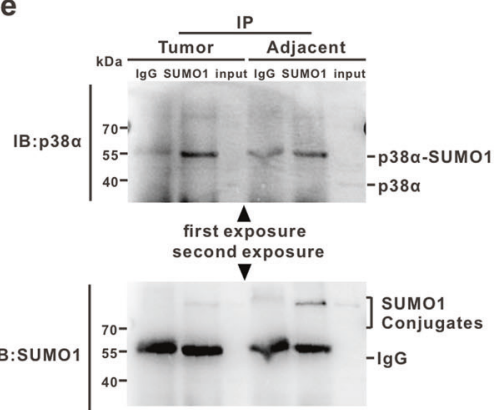

i
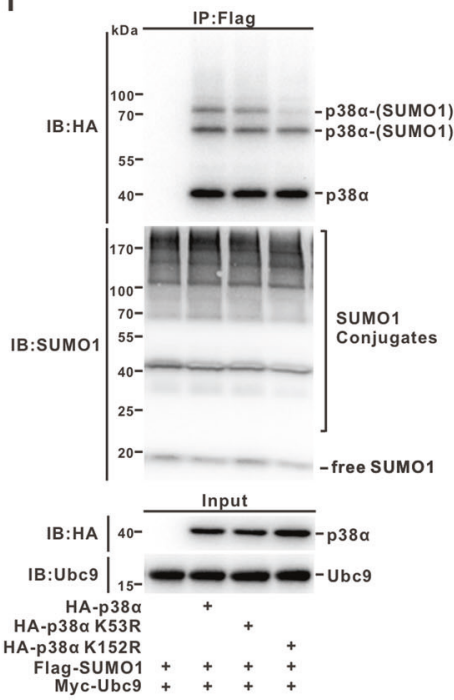

$\mathbf{f}$
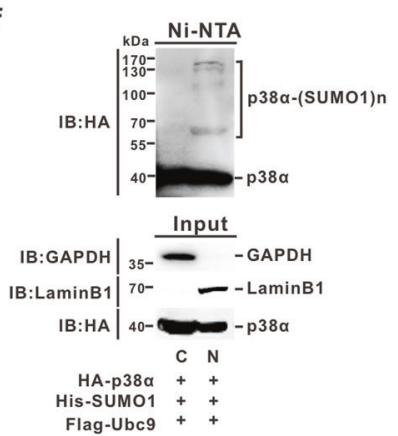

h

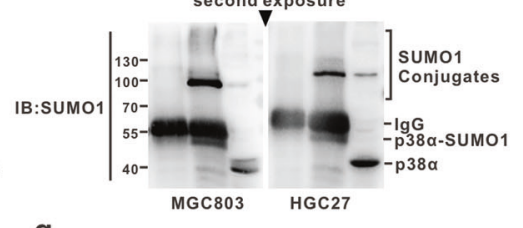

g

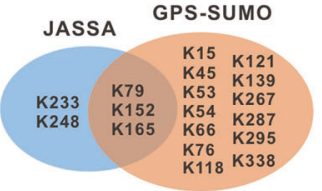

IB: $\mathrm{HA}$
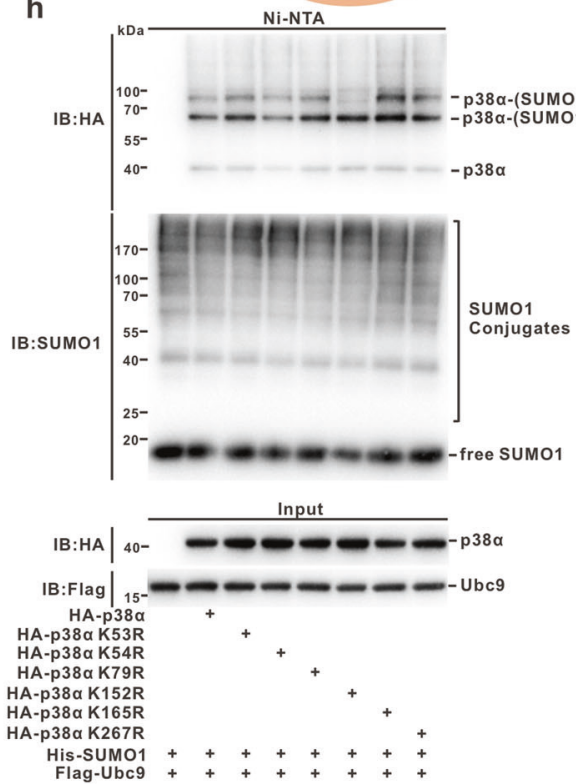

translocation [42]. Keeping this in mind, HA-p38awT and HAp38a $a^{\mathrm{D} 150 \mathrm{~A}}$ were introduced into HeLa cells, respectively, and a pulse-chase assay showed that the protein half-life of HAp38a $a^{\mathrm{D} 150 \mathrm{~A}}$ was significantly decreased as compared to that of HA-p38a ${ }^{\text {WT }}$ after treatment with cycloheximide (CHX) (Fig. 2e). 
Fig. 1 p38a can be SUMOylated at the major site K152 in the nucleus. a, b Lysis of HEK293T cells transfected with indicated plasmids was subjected to a subsequent $\mathrm{Ni}^{2+}$-NTA resin pull-down (a) or immunoprecipitated with mouse antiFLAG antibody (b), and exogenous SUMO1 modification of p38 $\alpha$ was immunoblotted with the indicated antibodies. c Plasmid pGEX-6p-1/p38 $\alpha$ was co-transfected with or without pE1/ E2/SUMO1 plasmid into E. coli BL21. Immunoblotting was performed with anti-SUMO1 antibody after GST pull-down assay; the same membrane was stripped and blotted with anti-GST antibody. d, e Endogenous SUMOylation on p38 $\alpha$ in GC cell lines (MGC803 and HGC27) (d), or in GC patient specimens (tumor and adjacent tissues) was analyzed with immunoprecipitation assay and detected with indicated antibodies. f After transfection with the indicated plasmids, HEK293T cells were extracted by the nuclear/cytosol fractionation kit, following which the lysates were pulled down by using the $\mathrm{Ni}^{2+}$-NTA resin and analyzed by immunoblotting. $\mathbf{g}$ The potential SUMOylation sites on p38 $\alpha$ predicted by JASSA and GPS-SUMO software are shown in the pie chart. $\boldsymbol{h}$ The construct of pEF-5HA/p38 $\alpha^{\text {WT }}$ or p38 $\alpha$ mutants was respectively co-transfected with His-SUMO1 into HEK293T cells. Cells were harvested $48 \mathrm{~h}$ after transfection and the lysates were analyzed by immunoblotting after pulling down with the $\mathrm{Ni}^{2+}-\mathrm{NTA}$ resin. $\mathbf{i}$ The construct, pEF-5HA/p38 $\alpha^{\mathrm{WT}}$ or p38 $\alpha-\mathrm{K} 152 \mathrm{R}$, was co-transfected with FLAG-SUMO1 into HEK293T cells. Cells were harvested at $48 \mathrm{~h}$ after transfection and lysates were analyzed by immunoblotting after immunoprecipitation with anti-FLAG antibody.

Strikingly, analysis of the nuclear-to-cytosolic ratio revealed that a fraction of HA-p38a ${ }^{\text {WT }}$ translocated to the nucleus, but HAp38a $\mathrm{a}^{\mathrm{D} 150 \mathrm{~A}}$ tended to localize in the cytoplasm (Fig. 2f). This result was corroborated by that of the immunofluorescence assay (Fig. 2g). To confirm that SUMOylation could affect the subcellular localization of p38a, HeLa cell lines with lower or higher SUMOylation conditions were constructed by depletion of Ubc9 or SENP1, respectively (Fig. S2a). Similar to the above results, the nuclearto-cytosolic ratio of endogenous p38a was decreased in HeLa-shUbc9 cells and increased in HeLa-shSENP1 compared with that in control cells (Fig. 2h). Consistently, the immunofluorescence assay also showed a similar phenomenon (Fig. 2i). Taken together, these data demonstrated that SUMOylation of p38a not only maintains protein stability but also ensures its nuclear translocation.

\section{p38a-SUMOylation facilitates GC metastasis in vitro and in vivo}

To investigate the cellular function performed by p38a-SUMOylation in GC, we established p38a, but not p38ß, knock-out cell lines MGC803/p38a $\mathrm{K}^{\mathrm{KO}}$ and HGC27/p38 $\mathrm{a}^{\mathrm{KO}}$ with the CRISPR-Cas9 system, and the resultant colonies were validated by Western blotting and genotyping (Fig. S2b-d). Next, we re-introduced p38a $\mathrm{a}^{\mathrm{WT}}$ or p38a $\mathrm{a}^{\mathrm{D} 150 \mathrm{~A}}$ into $\mathrm{MGC} 803 / \mathrm{p} 38 \mathrm{a}^{\mathrm{KO}}$ and $\mathrm{HGC} 27 / \mathrm{p} 38 \mathrm{a}^{\mathrm{KO}}$ cell lines by lentiviral infection (Fig. 3a). A series of phenotypic analyses revealed that $p 38 a^{\mathrm{D} 150 \mathrm{~A}}$, similar to $\mathrm{p} 38 \mathrm{a}$ knockout, significantly inhibited cell migration and invasion compared to $\mathrm{p} 38 \mathrm{a}^{\mathrm{WT}}$ in both MGC803 and HGC27 cells (Fig. 3b-e). However, there was no distinguished difference in cell proliferation between these cells (Fig. S2e, f). In the xenograft model, the specified cell lines were intravenously injected into nude mice, and the lungs of necropsied mice were collected for $\mathrm{H} \& \mathrm{E}$ staining and $\mathrm{IHC}$ detection with human vimentin antibody at 45 days post injection. p38a $\mathrm{KO}^{\mathrm{KO}}$ significantly decreased the tendency for pulmonary metastasis in GC cells, which was restored by re-introducing p38a $a^{\text {WT }}$, but not p38a $a^{\text {150A }}$ (Fig. 3f, g). Taken together, p38aSUMOylation can facilitate the metastasis processes of GC in vitro and in vivo, at least pulmonary metastasis.

\section{Positive feedback between p38a-SUMOylation and ROS facilitates metastasis of GC cells}

Since $p 38 a$ is a critical OS sensor, we wondered whether p38a SUMOylation could affect ROS generation in GC. Staining with either the DCFH-DA fluorescent probe or CellROX Orange reagent was performed in $\mathrm{HGC} 27$ cell lines. Both fluorescence microscopic observations and flow cytometry analysis revealed that desUMOylated p38a (p38a $a^{\text {D150A }}$ ) could not efficiently induce ROS in GC cells like p38a ${ }^{W T}$ (Fig. 4a-d). The primary ROS, $\mathrm{H}_{2} \mathrm{O}_{2}$, was also able to significantly promote the SUMOylation level of p38a (Fig. 4e, lanes 1-4). However, excess levels of $\mathrm{H}_{2} \mathrm{O}_{2}$ did not further enhance the SUMOylation process (Fig. $4 \mathrm{e}$, lane 5), which could be associated with an unknown selfprotective mechanism in tumor cells. In contrast, an antioxidant
$\mathrm{N}$-acetyl cysteine (NAC) was employed to confirm that ROS could positively induce SUMOylation event on p38a (Fig. 4f). According to the migration and invasion assay in HGC27 stable cell lines, NAC significantly suppressed the p38a-SUMOylationmediated metastatic properties of GC cells (Fig. $4 \mathrm{~g}$, h). Therefore, these data suggested that SUMOylation is critical for p38a to function as a sensor to generate ROS, and positive feedback between p38a-SUMOylation and ROS may contribute to the GC metastatic processes.

\section{MK2 activation is essential for p38a-SUMOylation to mediate OS response in GC cells}

To explore the mechanism through which p38a-SUMOylation mediates the OS response, which subsequently results in GC metastasis, we first focused on the closely related substrates of p38a: ATF2 and MK2. Western blotting demonstrated that the level of phosphorylated MK2 (pMK2, mainly at Thr222) in both MGC803 and HGC27 cells was significantly and completely recovered by re-introducing $\mathrm{p} 38 \mathrm{a}^{\mathrm{WT}}$ into $\mathrm{p} 38 \mathrm{a}^{\mathrm{KO}}$ cells, but not by introducing p38a $150 \mathrm{~A}$ (Fig. 5a). The levels of pATF2, as well as pp65, pSTAT1, and pSTAT3 (the other substrates of p38a), showed no significant difference between the p38a $\mathrm{D}^{150 \mathrm{~A}}$ and p38a ${ }^{\text {WT }}$ groups (Fig. 5a and Fig. S3a). Of note, the MK2 protein level was significantly decreased upon endogenous p38a knockout (Fig. 5a), which is probably due to the fact that p38a affects the transcription or protein stability of MK2 (not detected). Moreover, p38a was hyperactivated when p38a $a^{\text {D150A }}$ was re-introduced compared to p38a $a^{\text {WT }}$, but still could not efficiently activate MK2 (Fig. 5a) in both MGC803 and HGC27 cell lines. To determine the basis for altered pMK2 activation, we conducted a co-immunoprecipitation assay and found that the interaction between $\mathrm{p} 38 \mathrm{a}^{\mathrm{D} 150 \mathrm{~A}}$ and MK2 was significantly weakened (Fig. 5b). As mentioned above, the SUMOylation of p38a occurred in the nucleus (Fig. 1f), and de-SUMOylation would affect the translocation of p38a (Fig. $2 \mathrm{f}-\mathrm{i}$ ). To eliminate the possibility of translocation causing an alteration in interactions, the GST pull-down system in E. coli was employed. Results showed that the binding action between p38a and MK2 was largely dependent on the SUMOylation status of p38a (Fig. 5c).

As MK2 has been reported to play an important role in mediating ROS generation in neutrophils [43], we analyzed the role of MK2 in p38a-SUMOylation and OS response in GC. By blocking MK2 activity by MK2-IN-1 hydrochloride (MK2 specific inhibitor) (Fig. S3b), the extreme intracellular ROS generated in p38a ${ }^{\text {WT }}$ cells was reduced to almost the same level as that in p38a $a^{\mathrm{D} 150 A}$ or $\mathrm{p} 38 \mathrm{a}^{\mathrm{KO}}$ cells, as indicated by DCFH-DA probe staining (Fig. 5d) and flow cytometry analysis (Fig. 5e, f). In agreement with this result, CellROX staining and analysis also presented a similar outcome (Fig. $5 \mathrm{~g}-\mathrm{i}$ ). Taken together, these results elucidated that MK2 activation is essential for p38aSUMOylation, which further mediates the OS response in GC cells. 
a

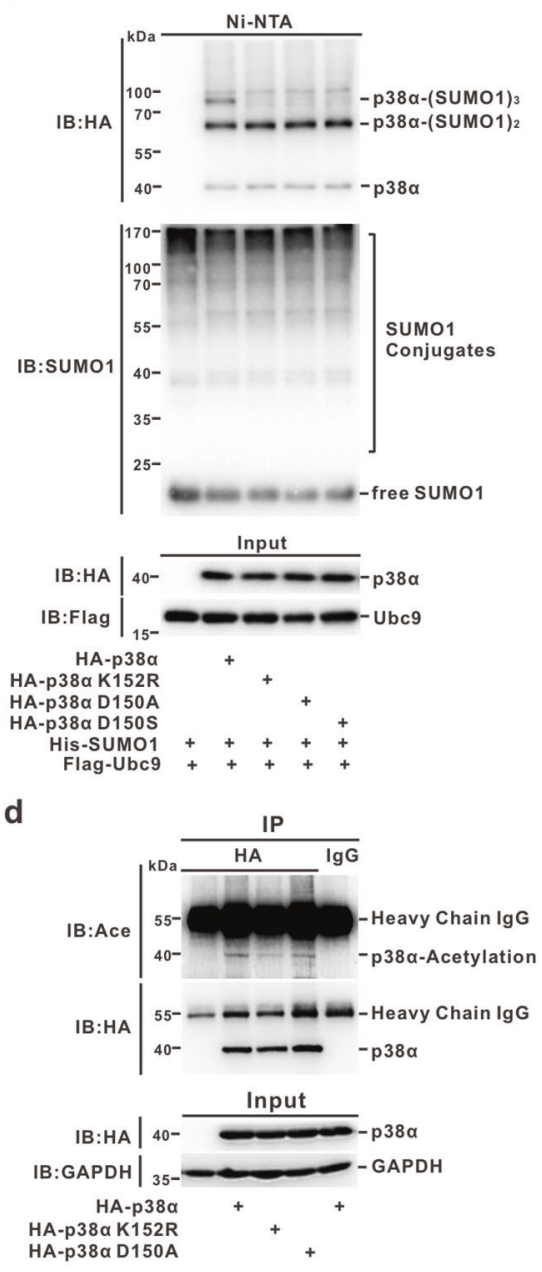

b

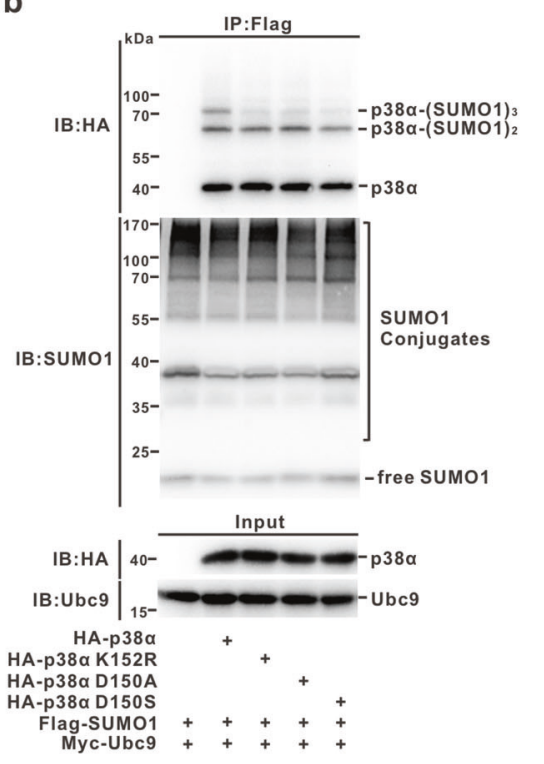

C

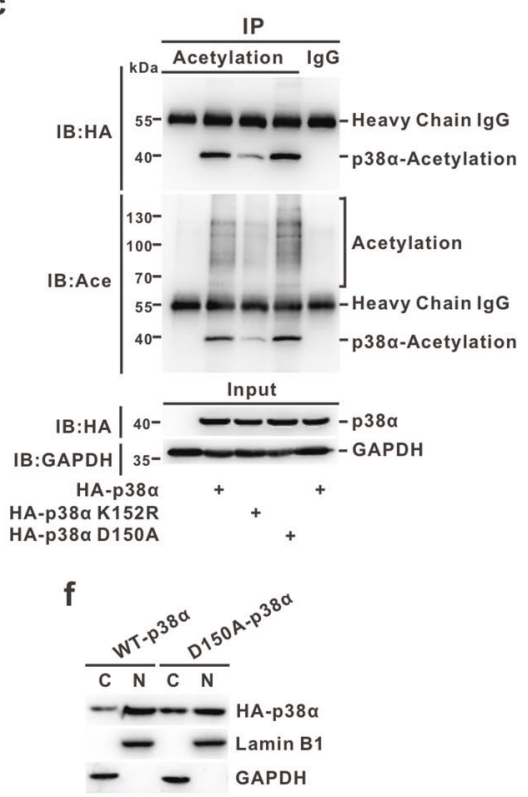

e

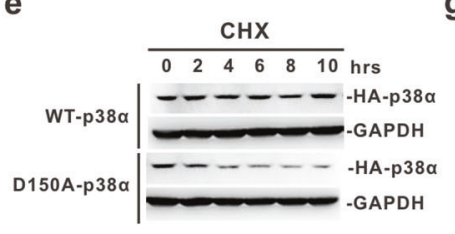

g
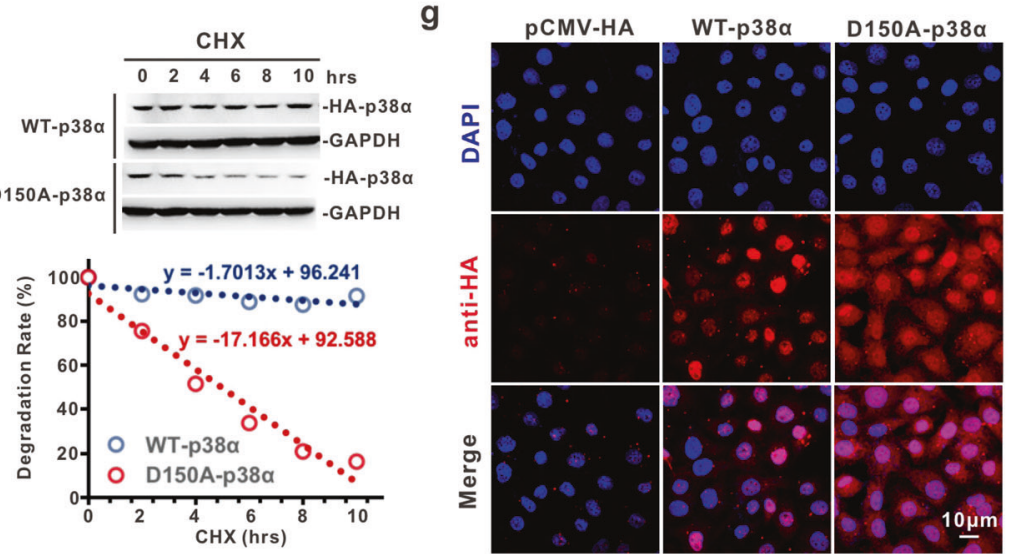

h

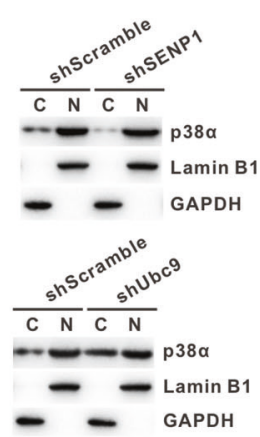

i



ROS can stabilize PIASxa to promote SUMOylation on p38a SUMOylation, similar to ubiquitylation, is a reversible modification mediated by E3 SUMO-protein transferases (PIASs) and deSUMOylation enzymes (SENPs) [42]. To determine how ROS affects p38a-SUMOylation, a total of five PIASs (PIAS1, PIASxa, PIASx $\beta$, PIAS3, PIAS4) were used for the GST pull-down assay and their ability to bind to p38a was analyzed. Results showed that PIASxa was more likely to interact with p38a (Fig. 6a). The endogenous interaction between PIASxa and p38a in HGC27 cells was also confirmed by the immunoprecipitation assay (Fig. 6b). To identify whether PIASxa could promote SUMOylation on p38a, the immunoprecipitation assay was performed. Results showed that p38a-SUMOylation was gradient enhanced following PIASxa transfection (Fig. $6 \mathrm{c}$ ). In contrast, ablation of PIASxa using siRNA reduced the SUMOylation level of p38a (Fig. 6d). All these indicated that PIASxa is an authentic and direct regulator of p38a.

Moreover, $\mathrm{H}_{2} \mathrm{O}_{2}$ or NAC enhanced the SUMOylation process on p38a (Fig. 4e, f). We next measured the protein level of PIASxa. 
Fig. 2 SUMOylation ensures protein stability and nuclear translocation of $\mathbf{p 3 8 a}$. $\mathbf{a}$, b The constructs containing $\mathrm{pEF}-5 \mathrm{HA} / \mathrm{p} 38 \alpha-\mathrm{WT}$, $-\mathrm{K} 152 \mathrm{R}$, -D150A, or -D150S were co-transfected with indicated plasmids into HEK293T cells. Cells were harvested at $48 \mathrm{~h}$ after transfection and the lysates were analyzed by immunoblotting after a $\mathrm{Ni}^{2+} \mathrm{NTA}$ resin pull-down (a) or immunoprecipitated with mouse anti-FLAG antibody (b). c, d Exogenous HA-p38 $\alpha-W T$, -K152R, or -D150A were transfected in HEK293T cells, and cell lysates were subjected to immunoprecipitation with antibody against ACE-lysine (c) or HA (d), immunoblotted with anti-HA or anti-ACE-lysine antibody, respectively. e Cells stably expressing HA-tagged p38 $\alpha$-WT or p38 $\alpha$-D150A were treated with $100 \mu \mathrm{M}$ CHX for time periods, and then cell lysates were subjected to immunoblotting analysis with the HA or GAPDH antibody (upper panel). The degradation rate was quantified as a line diagram (lower panel). $\mathbf{f}$, $\mathbf{g}$ HeLa cells transfected with HA-tagged p38 $\alpha$-WT or p38 $\alpha$-D150A were extracted by the nuclear/cytosol fractionation kit, and the lysates (total $50 \mu \mathrm{g} /$ sample) were subjected to immunoblotting with indicated antibodies (f) and immunofluorescence staining with primary mouse anti-HA antibody (1:200) and Alexa Fluor 568 labeled secondary antibody (1:200). The nuclei were counterstained with DAPI (g). h-i The stable HeLa cell lines with shRNAscramble, -SENP1 or -Ubc9 were extracted by the nuclear/cytosol fractionation kit, and lysates (total $50 \mu \mathrm{g} / \mathrm{sample}$ ) were subjected to immunoblotting with indicated antibodies (h) and immunofluorescence staining using the primary rabbit anti-p38 $\alpha$ antibody $(1: 200)$ and Alexa Fluor 488 labeled secondary antibody $(1: 200)$. Cell nuclei was counterstained with DAPI (i). The images were captured by Leica confocal microscopy, scale bar: $30 \mu \mathrm{m}(\mathbf{g}, \mathbf{i})$.

a

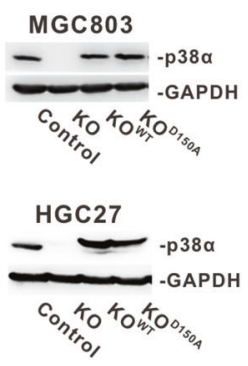

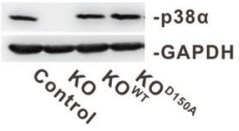

C

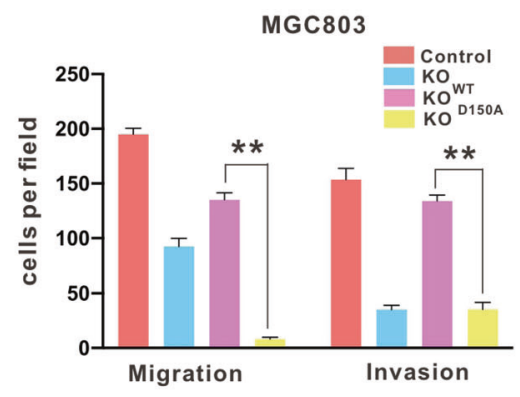

b

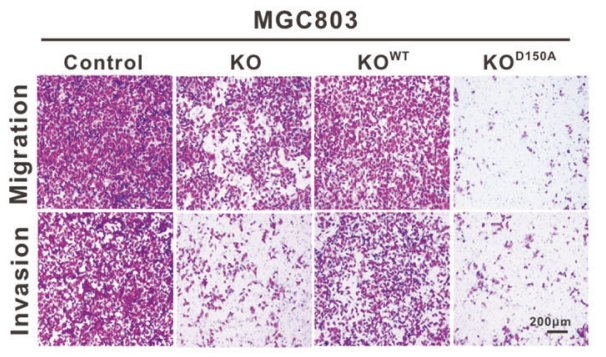

d

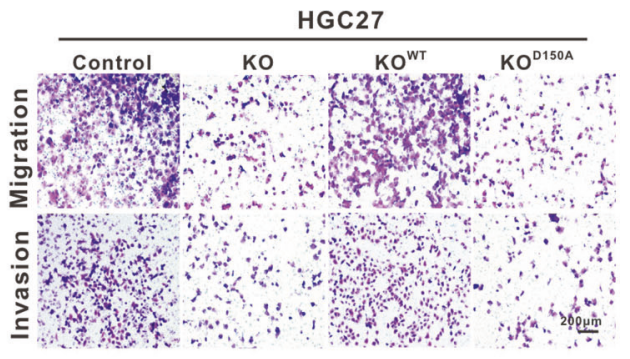

g

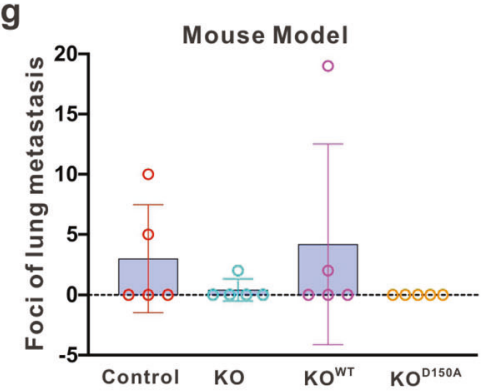

f

e

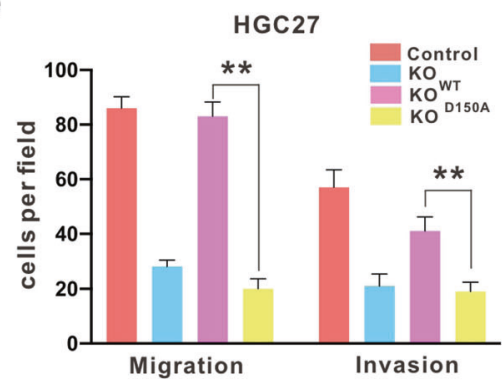

\section{.}


a
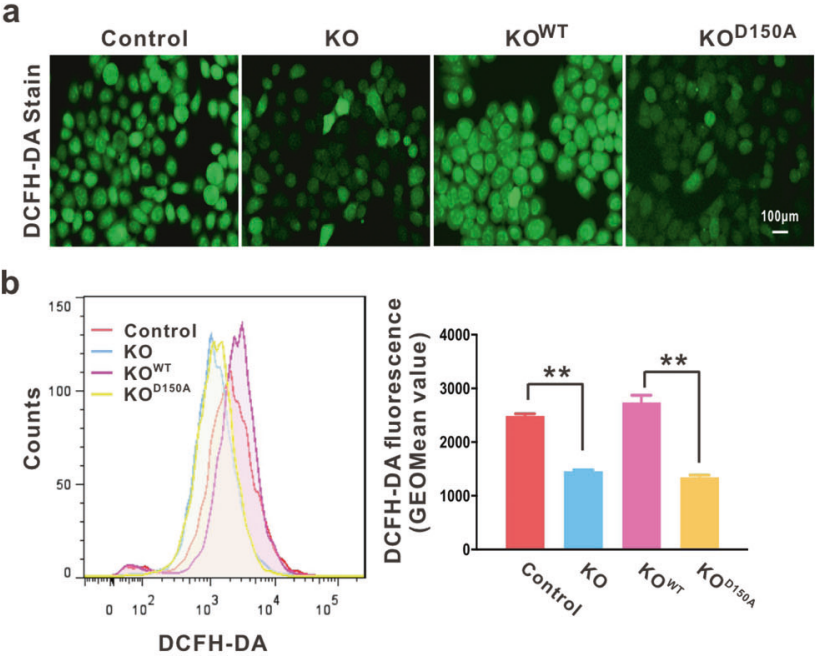

e
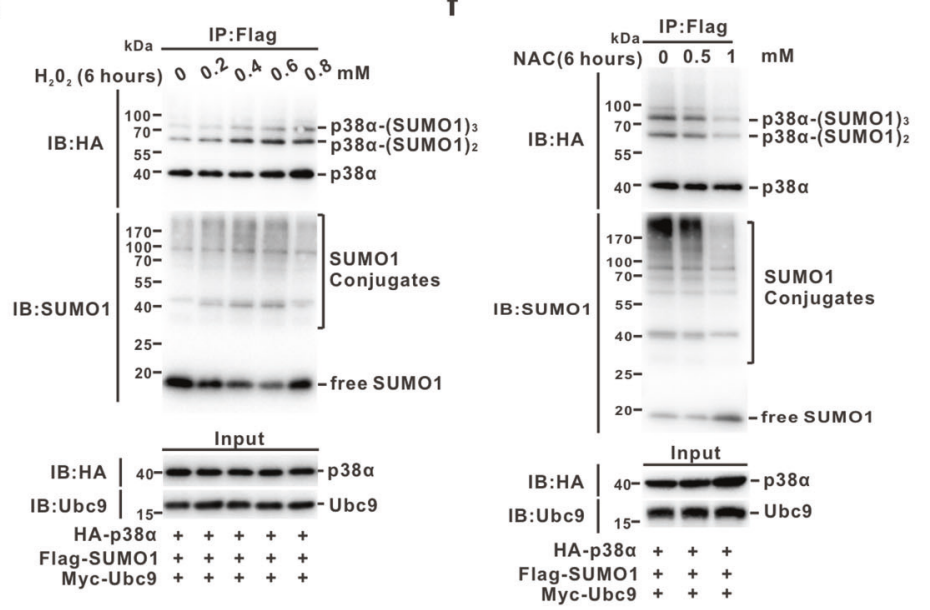

C

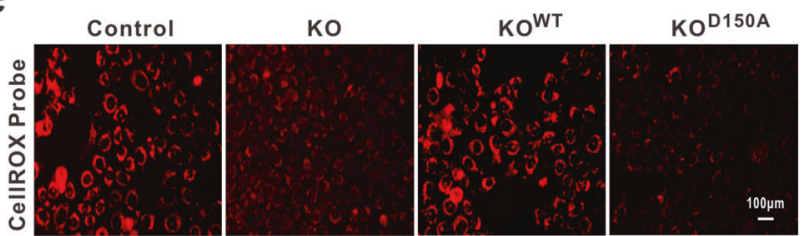

d
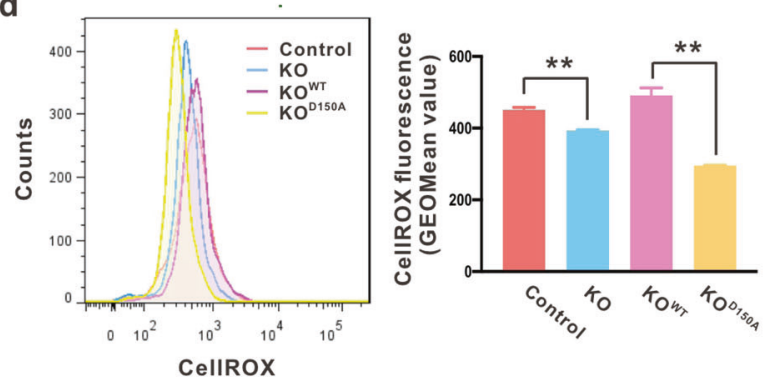

g

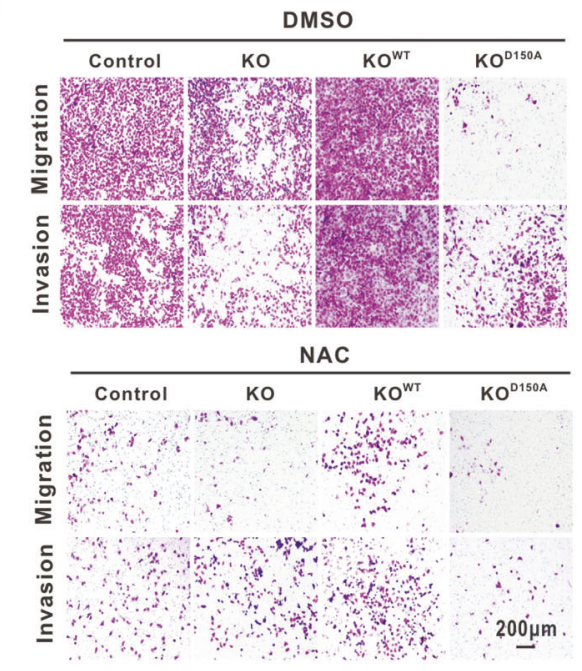

h
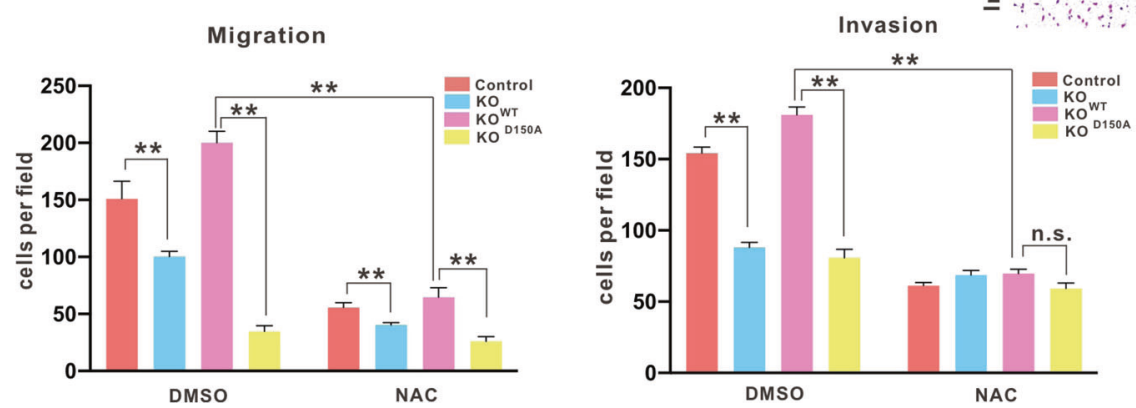

Fig. 4 Positive feedback between p38a-SUMOylation and ROS facilitates the metastatic property of GC cells. a-d ROS levels in HGC27/ MAPK $14^{\mathrm{KO}}$ cells with re-supplied $\mathrm{p} 38 \alpha^{\mathrm{WT}}$ and $\mathrm{p} 38 \alpha^{\mathrm{D} 150 \mathrm{~A}}$ were determined by DCFH-DA probe and CellRox Orange for fluorescence microscopic observation $(\mathbf{a}, \mathbf{c})$ and flow cytometry analysis $\left(\mathbf{b}, \mathbf{d}\right.$, left panels). The statistical data are presented as mean \pm S.D., ${ }^{* *} p<0.01(\mathbf{b}, \mathbf{d}$, right panels). e-f HEK293T cells co-transfected with FLAG-SUMO1, Myc-Ubc9, and HA-p38 $\alpha$-WT were treated with gradient concentrations of $\mathrm{H}_{2} \mathrm{O}_{2}$ or NAC for $6 \mathrm{~h}$, respectively, before harvesting. The cell lysates were immunoprecipitated with anti-FLAG antibody for SUMOylation assay and Western blotting. $\mathbf{g}-\mathbf{h}$ The indicated HGC27 stable cell lines were treated with DMSO or NAC (1 mM), respectively, for both migration and invasion assays $(\mathbf{g})$. The data are presented as the mean \pm S.D. ${ }^{* *} p<0.01$ (h).

Surprisingly, ROS could positively regulate PIASxa only at the protein level (Fig. 6e, f), but not at the mRNA level (Fig. S3c). Pulsechase assay revealed that $\mathrm{H}_{2} \mathrm{O}_{2}$ could significantly stabilize the PIASxa protein in GC cells (Fig. $6 \mathrm{~g}$ and Fig. S3d). Besides, MK2 activity was cis-regulated following $\mathrm{H}_{2} \mathrm{O}_{2}$ stimuli or NAC treatment in GC cells (Fig. 6e, f, Fig. S3e, f), which was consistent with that of HEK293T cells (Fig. S3g, h). Similarly, the endogenous p38aSUMOylation level was significantly enhanced by $\mathrm{H}_{2} \mathrm{O}_{2}$ (Fig. 6h). Clinically, the GEPIA database (http://gepia.cancer-pku.cn) showed that the expression of PIASx (including PIASxa and PIASx $\beta$ ) was significantly higher in gastric tumor samples than in normal tissues (Fig. 6i), and this finding was confirmed at the protein level, together with the activities of p38a and MK2, using fresh surgical specimens from patients (Fig. 6j). Moreover, the survival curve revealed that higher PIASx expression predicted poor survival probability, among overall, progression-free or post-progression (Fig. $6 \mathrm{k}-\mathrm{m}$ ). In conclusion, these data demonstrated that the oncogene PIASxa is stabilized by ROS and it promotes the 
a

b

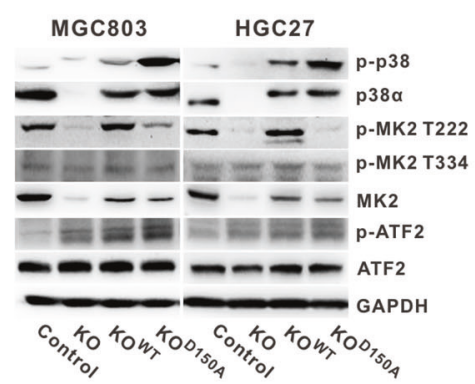

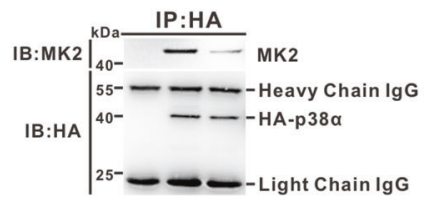

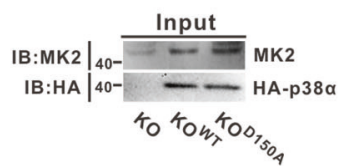

C

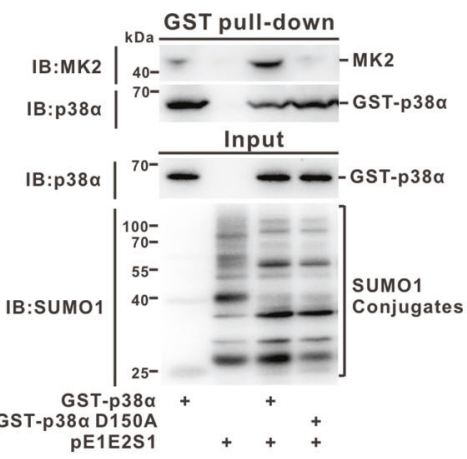

d
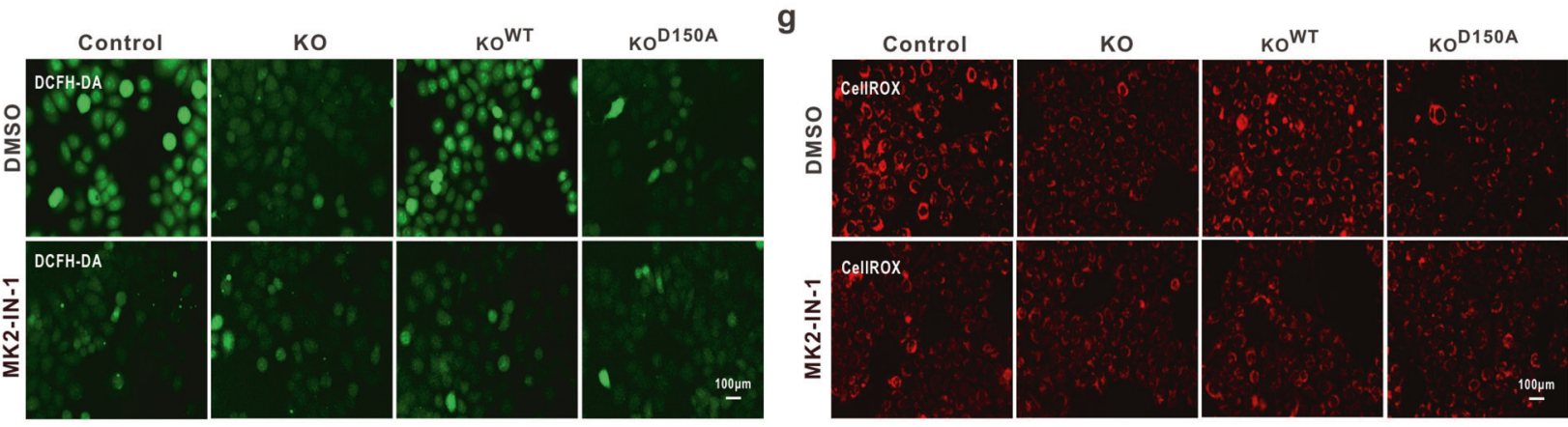

e
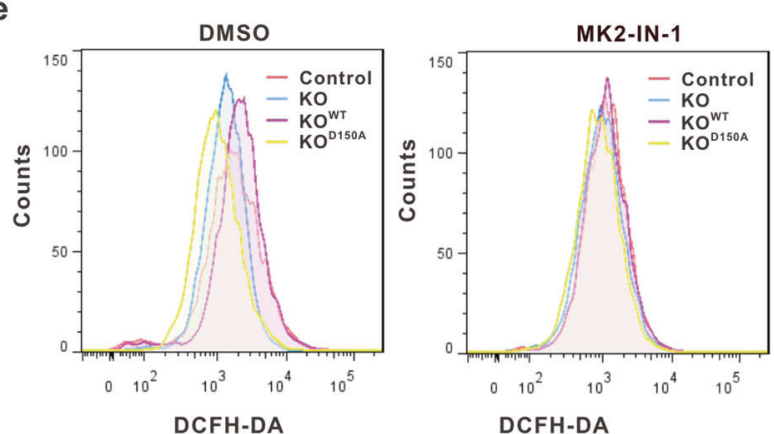

f

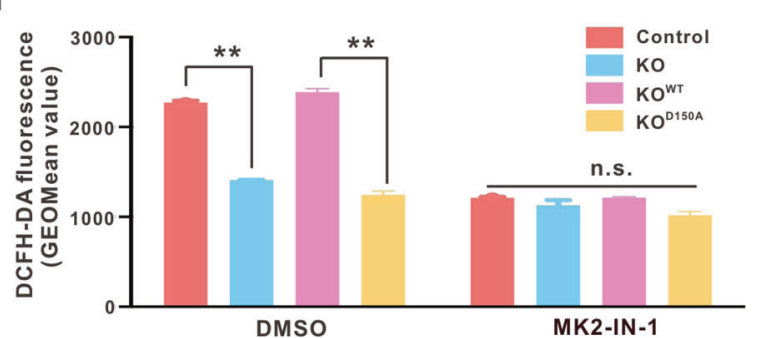

h
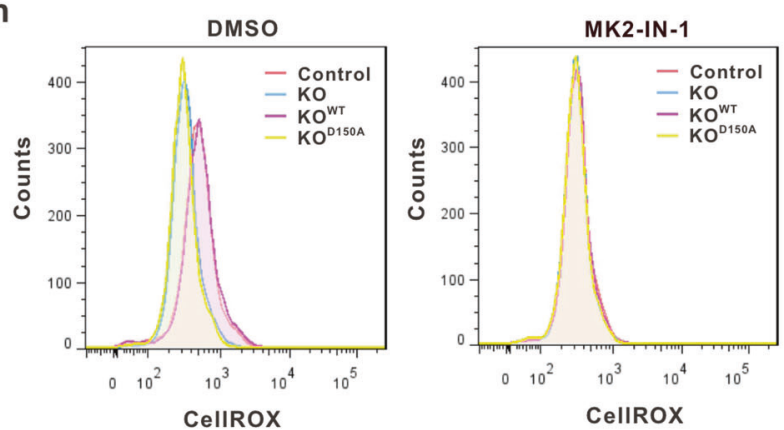

i

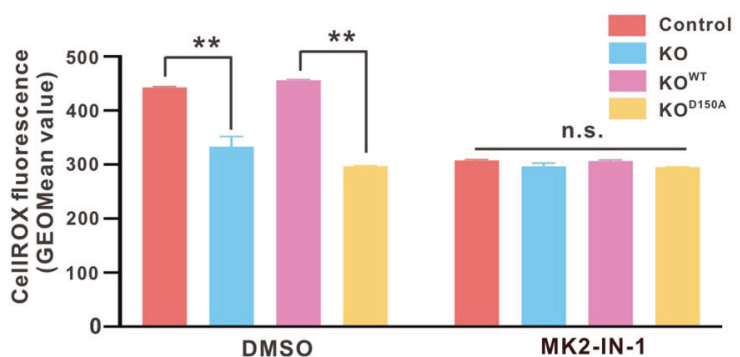

Fig. 5 MK2 activation is essential for p38a-SUMOylation to mediate OS response in GC cells. a p38-associated proteins were detected by

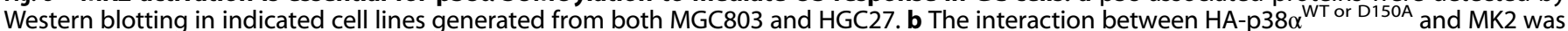
detected by co-immunoprecipitation analysis in MGC803/MAPK14 ${ }^{\mathrm{KO}}$ cells. c MGC803 cell lysates were incubated with purified GST-p38 $\alpha^{\text {WT }}$ or p38 $\alpha$ protein overnight at $4{ }^{\circ} \mathrm{C}$ and subjected to immunoblotting analysis. $\mathbf{d}-\mathbf{i}$ ROS levels in indicated HGC27 stable cell lines after treatment with MK2-IN-1 hydrochloride $(10 \mathrm{mM})$ for $24 \mathrm{~h}$ were determined by DCFH-DA probe (d-f) and CellRox Orange (g-i) for fluorescence microscopy $(\mathbf{d}, \mathbf{g})$ and flow cytometry analysis $(\mathbf{e}, \mathbf{h})$. The data are presented as mean \pm S.D. ${ }^{* *} p<0.01(\mathbf{f}, \mathbf{i})$.

SUMOylation process of $\mathrm{p} 38 \mathrm{a}$, which creates a form of positive feedback between ROS and the cis-axis PIASxa/p38a-SUMOylation/MK2 to facilitate the metastasis of GC (Fig. 7).

\section{DISCUSSION}

MAPK/p38 is an essential component of the MAPK signaling pathway and plays a critical role in the signaling cascades triggered by extra- or intra-cellular stimuli such as inflammatory cytokines or physical stress resulting in direct activation of transcription factors [2]. Prior to the present study, only three PTMs (i.e., phosphorylation, acetylation, and ubiquitination) of p38a have been reported, and each modification has a significant impact on the activity and function of p38a in cells (Fig. S4) [44]. Moreover, targeting MAPK/p38 has shown promising therapeutic potential in multiple cancers [44-46]. The poly-SUMO2 chain can noncovalently interact with p38 to enhance the nuclear transfer of pp38 upon H. pylori infection, but the function of SUMO-modified 
a
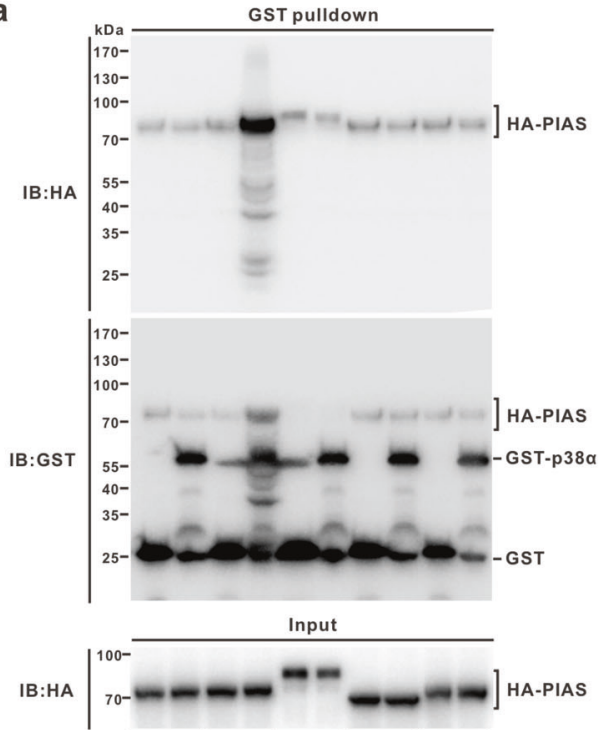

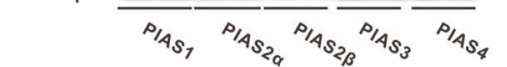

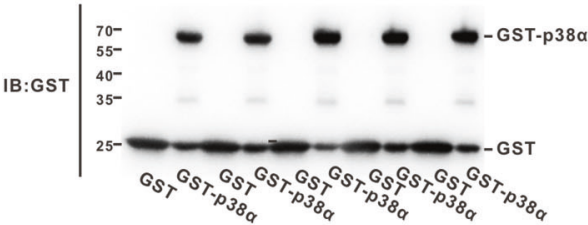

g

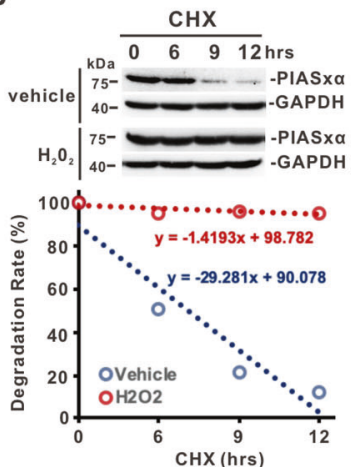

k

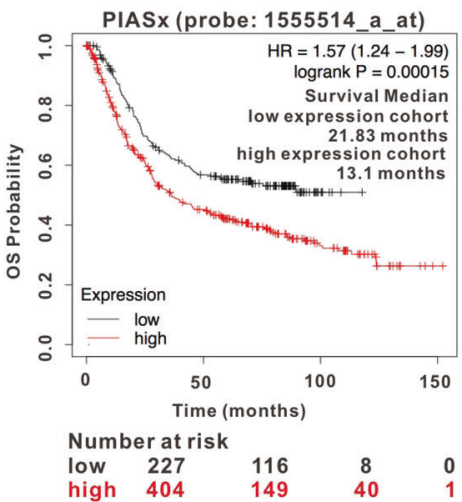

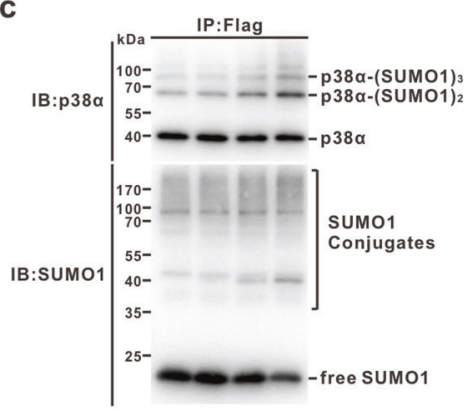

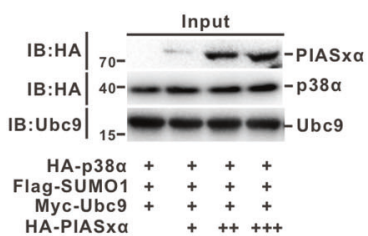

b

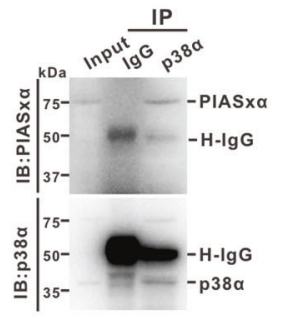

e
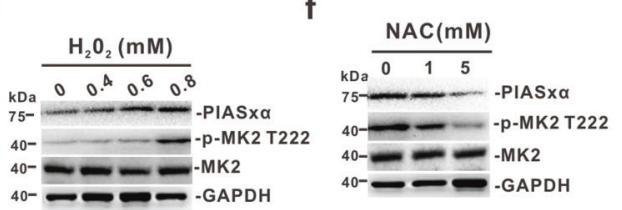

i

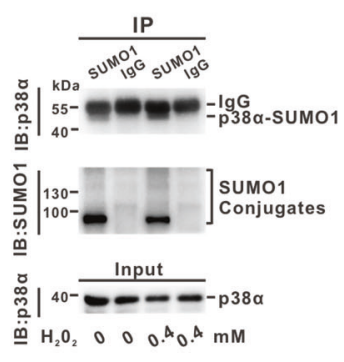

d
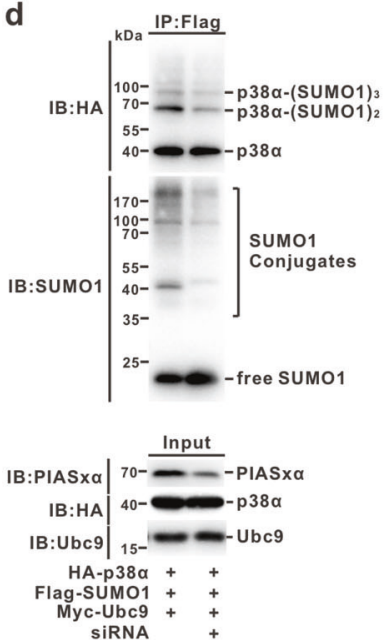

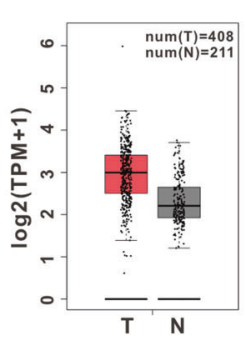

j

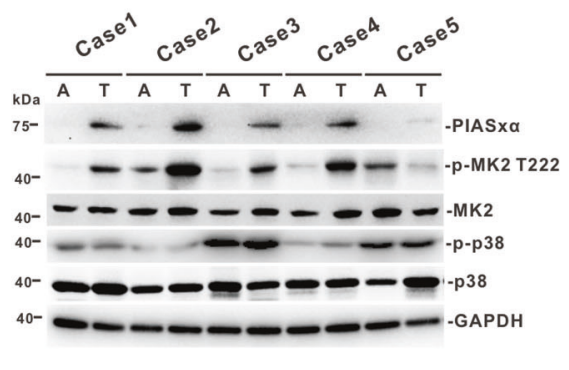

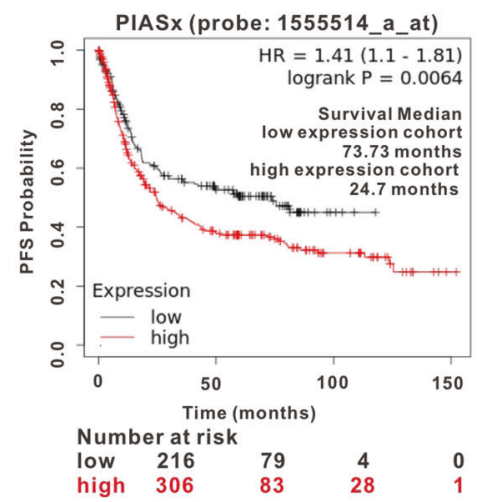

m

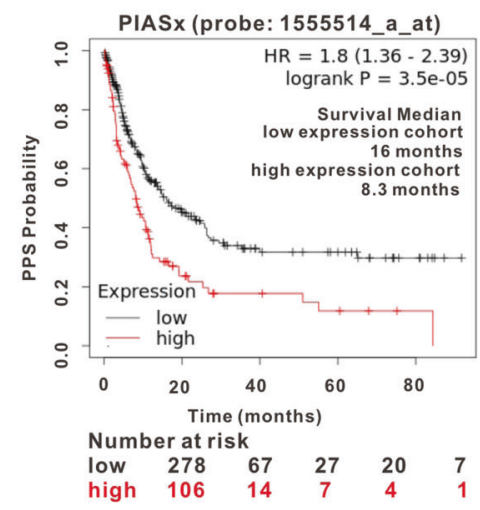

p38 is remains unclear [47]. Herein, we identified that SUMO1 modification mainly occurs at the $\mathrm{K} 152$ position of p38a. To exclude the impact of K152-acetylation in p38a, the alternative variant $\mathrm{p} 38 \mathrm{a}^{\mathrm{D} 150 \mathrm{~A}}$, which reduced SUMOylation without affecting the acetylation of p38a, was used for the functional illustration of
p38a-SUMOylation (Fig. 2a-d). According to our results, p38aSUMOylation can promote the metastasis of GC through activation of MK2, which induced ROS generation in vitro and in vivo (Figs. 3 and 5). Compared with $\mathrm{p} 38 \mathrm{a}^{\mathrm{KO}}$ cells, cells harboring SUMOylationdeficient p38a presented metastasis suppression phenotypes 
Fig. 6 ROS can stabilize PIASxa to promote p38a-SUMOylation. a HEK293T cells were transfected with HA-tagged PIASs for $48 \mathrm{~h}$, and cell lysates were incubated with purified GST-p38 $\alpha$ protein overnight at $4^{\circ} \mathrm{C}$ and subjected to immunoblotting analysis. $\mathbf{b}$ Immunoprecipitation was conducted to detect the endogenous interaction between PIASx $\alpha$ and p38 $\alpha$ in HGC27 cells. c HEK293T cells were co-transfected with FLAG-SUMO1, Myc-Ubc9, HA-p38 $\alpha$-WT, and gradient HAPIASx $\alpha$ for $48 \mathrm{~h}$; cell lysates were immunoprecipitated with anti-FLAG antibody for the SUMOylation assay. d HEK293T cells were transfected with FLAG-SUMO1, Myc-Ubc9, HA-p38 $\alpha$-WT with or without PIASx $\alpha$-siRNA, and cell lysates were immunoprecipitated for the SUMOylation assay. e, $\mathbf{f ~ H G C 2 7 ~ c e l l s ~ w e r e ~ t r e a t e d ~ w i t h ~ a ~ g r a d i e n t ~ c o n c e n t r a t i o n ~ o f ~} \mathrm{H}_{2} \mathrm{O}_{2}(\mathbf{e})$ or $\mathrm{NAC}(\mathbf{f})$ for $6 \mathrm{~h}$ before harvesting, and the cells were lysed for Western blotting. $\mathbf{g ~ H G C 2 7}$ cells stimulated by $\mathrm{H}_{2} \mathrm{O}_{2}$ or vehicle were treated with $50 \mu \mathrm{M}$ $\mathrm{CHX}$ for indicated times and cell lysates were subjected to immunoblotting (upper panel). The degradation rate is represented as a line diagram (lower panel). $\mathbf{h ~ H G C 2 7}$ cells were treated with or without $\mathrm{H}_{2} \mathrm{O}_{2}(0.8 \mathrm{mM})$ for $6 \mathrm{~h}$ and were harvested for detecting the SUMOylation level of endogenous p38 $\alpha$ by immunoprecipitation. i GEPIA online database was used to show the mRNA level of PIASx in GC tissue and normal gastric tissues (T: tumor; N: normal). $\mathbf{j}$ Immunoblotting for determination of PIASx $\alpha$, pp38, and pMK2 levels in 5 pairs of GC tissues and adjacent normal tissues (A: adjacent normal gastric tissues; T: GC tissues). $\mathbf{k}-\mathbf{m}$ The survival probabilities were predicted by the online database. OS: overall survival (k), PFS: progression-free survival (I), or PPS: post-progression survival (m). Patients with high or low PIASx (PIASx $\alpha$ and PIASx $\beta$ ) expression are indicated in red or black line, respectively. HR hazard ratio. The $p$-value was calculated by a log-rank test.

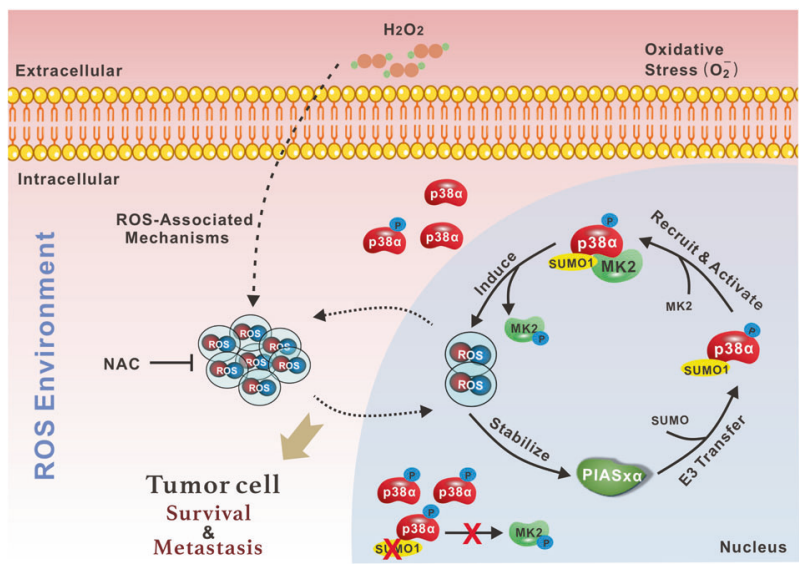

Fig. 7 Graphical abstract. ROS, as a double-edged sword, possess both pro- and anti-tumorigenesis properties, which depends primarily on intracellular ROS level and tumor type. In gastric cancer, the oxidative stress sensor p38 $\alpha$ can undergo SUMO1 modification to facilitate its nuclear translocation, and p38 $\alpha$ SUMOylation is essential for the activation of MK2 in the nucleus, thereby mediating ROS generation in cells. Meanwhile, an appropriate amount of ROS can stabilize SUMO E3 ligase PIASx $\alpha$ and further promote the SUMOylation of $p 38 \alpha$, thus forming a positive feedback between ROS and cis-axis PIASx $\alpha /$ p38 $\alpha$-SUMOylation/MK2. This specific regulatory mechanism is exploited by gastric cancer cells to maintain initial tumor cell survival and further metastasis.

(Figs. $3 b-g$ and $4 g, h$ ), as well as a reduced capability for producing ROS (Figs. $4 \mathrm{a}-\mathrm{d}$ and $5 \mathrm{~d}-\mathrm{i}$ ). We also observed that ROS can promote the SUMOylation of p38a (Fig. 4e, f), creating a positive feedback loop.

Several studies have shown that the p38/MK2 pathway is closely related to the OS response [43, 48, 49]; however, we revealed that SUMOylation is required by p38a to activate MK2 in the nucleus (Fig. $5 \mathrm{a}-\mathrm{C}$ ) and to generate ROS in GC cells (Fig. $5 \mathrm{~d}-\mathrm{i}$ ), and this requirement appears to be independent of p38 phosphorylation levels. SUMOylation-deficient p38a was unable to enter into or remain in the nucleus for MK2 activation, despite p38a hyperactivation (Fig. 5a, lane 4 in each panel). By contrast, the difference in ROS levels under p38a-SUMOylation and deSUMOylation could be abolished by an MK2 inhibitor (Fig. $5 \mathrm{~d}-\mathrm{i}$ ). Hence, p38a-SUMOylation-mediated ROS generation is largely dependent on MK2 activity.

This study also explored how ROS affects the SUMOylation of p38a. ROS could significantly stabilize PIASxa (Fig. 6e-g) and specifically interact with $\mathrm{p} 38 \mathrm{a}$ in vivo and in vitro to promote its SUMOylation (Fig. 6a-d). PIASxa and PIASx $\beta$ share the same promoter and are closely associated with the MAPK/p38 signaling pathway $[50,51]$. MAPK/p38 activation can stabilize PIASx $\beta$ and promote its transcription [50]. The phosphorylation of PIASxa mediated by $p 38 \beta 2$ is deemed to dampen the activation of Elk1 by maintaining Elk1 SUMOylation and HDAC2 recruitment under anisomycin stimulation [50]. However, we observed that the stability of PIASxa is also related to the ROS level and positively correlates with the status of MK2 phosphorylation or p38aSUMOylation level (Fig. 6e, f, h).

To be mentioned, there still are some shortcomings in this work, such as that the un-SUMOylated HA-p38a in control samples was prone to attach to the agarose or Ni-NTA resin non-specifically according to the Western blot analysis even though we have tried our best to solve this issue through optimizing the lysis and wash buffer (Figs. 1a, b and 2a, b). More importantly, the residual deSUMOylation enzymes, especially in the non-denatured RIPA buffer for immunoprecipitation assay, could inevitably remove the SUMO1 conjugates from the SUMOylated-p38a and lead to the detectable events of un-modified species during the experimental operation (Figs. 1a, b, i, 2a, b, 4e, f, and 6c, d). In addition, we have not succeeded in the reciprocal immunoprecipitation with $\mathrm{HA}$ antibody for p38a-SUMOylation assay, which could be affected by the poor immunoprecipitation efficiency of antibody for the HAp38a-SUMOylation species (Fig. 1b).

In summary, we revealed that $\mathrm{p} 38 \mathrm{a}$ can be utilized by GC cells for PIASxa-mediated SUMOylation in the nucleus to enhance MK2 activity and generate moderate levels of ROS for survival and to facilitate metastasis. Additionally, ROS can stabilize PIASxa to create a positive feedback loop between ROS and the cis-axis of PIASxa/p38a-SUMOylation/MK2. However, how ROS regulates the stability of PIASxa still need to be further researched, in which the specific ubiquitin E3 ligase or deubiquitinase could be the key regulators of PIASxa upon OS in GC. Beyond that, there must be certain unknown negative feedback mechanism to restrain PIASxa/p38a-SUMOylation/MK2 signal under the high ROS environment to resist the damage of high ROS on tumor cells. Here, our findings provide novel insights into the role of p38a in GC and provide potential therapeutic strategies for GC treatment, as targeting PIASxa, MK2, or a specific peptide region of p38a may resolve aberrant intracellular OS in GC cells and impede metastasis. Importantly, more attention should be paid to investigate the precise regulation mechanism of oxidative stress in various tumor diseases for therapeutic strategy making.

\section{AVAILABILITY OF DATA AND MATERIALS}

All the datasets generated and/or analyzed during this present study are available from the corresponding author on reasonable request.

\section{REFERENCES}

1. Hunter T. Protein kinases and phosphatases: the yin and yang of protein phosphorylation and signaling. Cell. 1995;80:225-36.

2. Ono $\mathrm{K}, \mathrm{Han} \mathrm{JH}$. The p38 signal transduction pathway-activation and function. Cell Signal. 2000;12:1-13. 
3. Johnson GL, Lapadat R. Mitogen-activated protein kinase pathways mediated by ERK, JNK, and p38 protein kinases. Science. 2002;298:1911-2.

4. Brancho D, Tanaka N, Jaeschke A, Ventura JJ, Kelkar N, Tanaka Y, et al. Mechanism of p38 MAP kinase activation in vivo. Genes Dev. 2003;17:1969-78.

5. Rezatabar S, Karimian A, Rameshknia V, Parsian H, Majidinia M, Kopi TA, et al. RAS/ MAPK signaling functions in oxidative stress, DNA damage response and cancer progression. J Cell Physiol 2019.

6. Ito K, Hirao A, Arai F, Takubo K, Matsuoka S, Miyamoto K, et al. Reactive oxygen species act through p38 MAPK to limit the lifespan of hematopoietic stem cells. Nat Med. 2006;12:446-51.

7. Zlotorynski E. DNA damage puts p38 under the UV light. Nat Rev Mol Cell Biol. 2018;19:279.

8. Hayes JD, Dinkova-Kostova AT, Tew KD. Oxidative stress in cancer. Cancer Cell. 2020;38:167-97.

9. Dolado I, Swat A, Ajenjo N, De Vita G, Cuadrado A, Nebreda AR. p38alpha MAP kinase as a sensor of reactive oxygen species in tumorigenesis. Cancer Cell. 2007;11:191-205.

10. Smyth EC, Nilsson M, Grabsch HI, van Grieken NC, Lordick F. Gastric cancer. Lancet. 2020;396:635-48.

11. Rugge M. Gastric cancer risk: between genetics and lifestyle. lancet Oncol. 2020;21:1258-60.

12. Van Cutsem E, Sagaert X, Topal B, Haustermans K, Prenen H. Gastric cancer. Lancet. 2016;388:2654-64.

13. Blair VR, McLeod M, Carneiro F, Coit DG, D'Addario JL, van Dieren JM, et al. Hereditary diffuse gastric cancer: updated clinical practice guidelines. Lancet Oncol. 2020;21:e386-e397.

14. Huang $Q$, Lan F, Wang $X, Y u$ Y, Ouyang $X$, Zheng F, et al. IL-1beta-induced activation of $\mathrm{p} 38$ promotes metastasis in gastric adenocarcinoma via upregulation of AP-1/c-fos, MMP2 and MMP9. Mol Cancer. 2014;13:18.

15. Grabsch HI, Tan P. Gastric cancer pathology and underlying molecular mechanisms. Dig Surg. 2013;30:150-8.

16. Bhattacharyya A, Chattopadhyay R, Mitra S, Crowe SE. Oxidative stress: an essential factor in the pathogenesis of gastrointestinal mucosal diseases. Physiol Rev. 2014;94:329-54.

17. Sies H, Berndt C, Jones DP. Oxidative stress. Annu Rev Biochem. 2017;86:715-48.

18. Ge W, Zhao K, Wang X, Li H, Yu M, He M, et al. iASPP is an antioxidative factor and drives cancer growth and drug resistance by competing with Nrf2 for Keap1 binding. Cancer Cell. 2017;32:561-73 e566.

19. Lu YX, Ju HQ, Liu ZX, Chen DL, Wang Y, Zhao Q, et al. ME1 regulates NADPH homeostasis to promote gastric cancer growth and metastasis. Cancer Res. 2018;78:1972-85.

20. Li S, Zhuang Z, Wu T, Lin JC, Liu ZX, Zhou LF, et al. Nicotinamide nucleotide transhydrogenase-mediated redox homeostasis promotes tumor growth and metastasis in gastric cancer. Redox Biol. 2018;18:246-55.

21. Chen $X$, Chen X, Zhang X, Wang L, Cao P, Rajamanickam V, et al. Curcuminoid B63 induces ROS-mediated paraptosis-like cell death by targeting TrxR1 in gastric cells. Redox Biol. 2019;21:101061.

22. Finkel T, Holbrook NJ. Oxidants, oxidative stress and the biology of ageing. Nature. 2000;408:239-47.

23. Demple B, Amabile-Cuevas CF. Redox redux: the control of oxidative stress responses. Cell. 1991;67:837-9.

24. Harris IS, DeNicola GM. The complex interplay between antioxidants and ROS in cancer. Trends Cell Biol. 2020;30:440-51.

25. Matsui R, Ferran B, Oh A, Croteau D, Shao D, Han J, et al. Redox regulation via Glutaredoxin-1 and Protein S-Glutathionylation. Antioxid Redox Signal. 2020;32:677-700.

26. McLoughlin MR, Orlicky DJ, Prigge JR, Krishna P, Talago EA, Cavigli IR, et al. TrxR1, Gsr, and oxidative stress determine hepatocellular carcinoma malignancy. Proc Natl Acad Sci USA. 2019;116:11408-17.

27. Huang $X$, He $C$, Hua $X, \operatorname{Kan} A$, Mao $Y$, Sun $S$, et al. Oxidative stress induces monocyte-to-myofibroblast transdifferentiation through p38 in pancreatic ductal adenocarcinoma. Clin Transl Med. 2020;10:e41.

28. Zhong W, Zhu H, Sheng F, Tian Y, Zhou J, Chen Y, et al. Activation of the MAPK11/ $12 / 13 / 14$ (p38 MAPK) pathway regulates the transcription of autophagy genes in response to oxidative stress induced by a novel copper complex in HeLa cells. Autophagy. 2014;10:1285-300.

29. Hay RT. SUMO: a history of modification. Mol cell. 2005;18:1-12.

30. Seeler JS, Dejean A. SUMO and the robustness of cancer. Nat Rev Cancer. 2017;17:184-97.

31. Chen C, Zhu C, Huang J, Zhao X, Deng R, Zhang H, et al. SUMOylation of TARBP2 regulates miRNA/siRNA efficiency. Nat Commun. 2015;6:8899.

32. Zhu C, Chen $C$, Chen R, Deng R, Zhao X, Zhang H, et al. K259-SUMOylation of DGCR8 promoted by p14ARF exerts a tumor-suppressive function. J Mol Cell Biol. 2016:8:456-8.

33. Barry $\mathrm{R}$, John SW, Liccardi G, Tenev $\mathrm{T}$, Jaco I, Chen $\mathrm{CH}$, et al. SUMO-mediated regulation of NLRP3 modulates inflammasome activity. Nat Commun. 2018;9:3001.
34. Stankovic-Valentin N, Drzewicka K, Konig C, Schiebel E, Melchior F. Redox regulation of SUMO enzymes is required for ATM activity and survival in oxidative stress. EMBO J. 2016;35:1312-29.

35. Cheng J, Kang X, Zhang S, Yeh ET. SUMO-specific protease 1 is essential for stabilization of HIF1alpha during hypoxia. Cell. 2007;131:584-95.

36. de la Vega L, Grishina I, Moreno R, Kruger M, Braun T, Schmitz ML. A redoxregulated SUMO/acetylation switch of HIPK2 controls the survival threshold to oxidative stress. Mol Cell. 2012;46:472-83.

37. Graves JD, Lee YJ, Liu K, Li G, Lin FT, Lin WC. E2F1 sumoylation as a protective cellular mechanism in oxidative stress response. Proc Natl Acad Sci USA 2020;117:14958-69.

38. Lu W, Wang Q, Xu C, Yuan H, Fan Q, Chen B, et al. SUMOylation is essential for Sirt2 tumor-suppressor function in neuroblastoma. Neoplasia. 2021;23:129-39.

39. Huang J, Yan J, Zhang J, Zhu S, Wang Y, Shi T, et al. SUMO1 modification of PTEN regulates tumorigenesis by controlling its association with the plasma membrane. Nat Commun. 2012;3:911.

40. Barysch SV, Dittner C, Flotho A, Becker J, Melchior F. Identification and analysis of endogenous SUMO1 and SUMO2/3 targets in mammalian cells and tissues using monoclonal antibodies. Nat Protoc. 2014;9:896-909.

41. Pillai VB, Sundaresan NR, Samant SA, Wolfgeher D, Trivedi CM, Gupta MP. Acetylation of a conserved lysine residue in the ATP binding pocket of p38 augments its kinase activity during hypertrophy of cardiomyocytes. Mol Cell Biol. 2011;31:2349-63.

42. Creton S, Jentsch S. SnapShot: the SUMO system. Cell. 2010;143:848-848 e841.

43. Sun L, Wu Q, Nie Y, Cheng N, Wang R, Wang G, et al. A role for MK2 in enhancing neutrophil-derived ROS production and aggravating liver ischemia/reperfusion injury. Front Immunol. 2018;9:2610.

44. Zou X, Blank M. Targeting p38 MAP kinase signaling in cancer through posttranslational modifications. Cancer Lett. 2017;384:19-26.

45. Lee MR, Dominguez C. MAP kinase p38 inhibitors: clinical results and an intimate look at their interactions with p38alpha protein. Curr Med Chem. 2005;12:2979-94.

46. Rudalska R, Dauch D, Longerich T, McJunkin K, Wuestefeld T, Kang TW, et al. In vivo RNAi screening identifies a mechanism of sorafenib resistance in liver cancer. Nat Med. 2014;20:1138-46.

47. Wang PY, Hsu PI, Wu DC, Chen TC, Jarman AP, Powell LM, et al. SUMOs mediate the nuclear transfer of p38 and p-p38 during Helicobacter pylori infection. Int J Mol Sci. 2018;19:9.

48. Trempolec N, Munoz JP, Slobodnyuk K, Marin S, Cascante M, Zorzano A, et al Induction of oxidative metabolism by the p38alpha/MK2 pathway. Sci Rep. 2017;7:11367.

49. Ashraf $M I$, Ebner $M$, Wallner $C$, Haller $M$, Khalid $S$, Schwelberger $H$, et al. A p38MAPK/MK2 signaling pathway leading to redox stress, cell death and ischemia/reperfusion injury. Cell Commun Signal. 2014;12:6.

50. Yang SH, Sharrocks AD. PIASxalpha differentially regulates the amplitudes of transcriptional responses following activation of the ERK and p38 MAPK pathways. Mol Cell. 2006;22:477-87.

51. Ohshima T, Shimotohno K. Transforming growth factor-beta-mediated signaling via the p38 MAP kinase pathway activates Smad-dependent transcription through SUMO-1 modification of Smad4. J Biol Chem. 2003;278:50833-42.

\section{ACKNOWLEDGEMENTS}

We thank Dr. Jianxiu Yu and Dr. Jian Huang in Shanghai Jiao Tong University School of Medicine for providing His-SUMO1, EBG-SENP1, Myc-UBC9, HA-PIAS1, -PIASxa, -PIASx $\beta$, -PIAS3, and -PIAS4 plasmids.

\section{AUTHOR CONTRIBUTIONS}

M.X., Y.Z., and M.L. designed and guided the study, and Q.W. carried out most of the experiments and data collection; Q.W. and C.X. performed ROS quantification, SUMOylation assay, H \& E staining and IHC. Q.W., B.C., and R.C performed cloning, mutagenesis, and viral infection assays. Q.W., C.X., Q.F., and X.Z. performed animal experiments; Q.F., Y.Z., and H.Y. collected the clinical data and information; M.X., Q.W., and C.X. wrote and edited the manuscript.

\section{FUNDING}

We acknowledge the financial support from National Natural Science Foundation of China grants 81672708 (to M.X.), 81802563 (to H.Y.), Shanghai International Cooperation Foundation 18410720200 (to Y.Z.), Fundamental Research Program Funding of Ninth People's Hospital affiliated to Shanghai Jiao Tong University School of Medicine to Q.W. (JYZZ045), and C.X. (JYZZ074). 
12

\section{ETHICS APPROVAL}

This study involved patient samples and animals that all were approved by Committee of Experiments Research at Shanghai Ninth People's Hospital, Shanghai Jiao Tong University School of Medicine.

\section{COMPETING INTERESTS}

The authors declare no competing interests.

\section{ADDITIONAL INFORMATION}

Supplementary information The online version contains supplementary material available at https://doi.org/10.1038/s41419-021-04302-6.

Correspondence and requests for materials should be addressed to Yanjie Zhang, Moubin Lin or Ming Xu.

Reprints and permission information is available at http://www.nature.com/ reprints
Publisher's note Springer Nature remains neutral with regard to jurisdictional claims in published maps and institutional affiliations. (c) (i) Open Access This article is licensed under a Creative Commons Attribution 4.0 International License, which permits use, sharing, adaptation, distribution and reproduction in any medium or format, as long as you give appropriate credit to the original author(s) and the source, provide a link to the Creative Commons license, and indicate if changes were made. The images or other third party material in this article are included in the article's Creative Commons license, unless indicated otherwise in a credit line to the material. If material is not included in the article's Creative Commons license and your intended use is not permitted by statutory regulation or exceeds the permitted use, you will need to obtain permission directly from the copyright holder. To view a copy of this license, visit http://creativecommons. org/licenses/by/4.0/.

(c) The Author(s) 2021 